\title{
Diferentes vías de lipólisis como posibles opciones terapéuticas de la obesidad
}

\author{
Different routes of lipolysis as possible new \\ therapeutic approaches of obesity
}

\author{
Diana Vargas, luis Celis, Fernando Lizcano • Bogotá, D.C. (Colombia)
}

\section{Resumen}

Objetivo: la obesidad ha aumentado en todo el mundo, no obstante existen pocas opciones terapéuticas novedosas y la inclinación a actividades cruentas para la terapia de obesidad y diabetes mellitus 2 no dejan de ser un riesgo. Con el fin de valorar el efecto de compuestos sobre la actividad de la célula grasa, estudiamos en forma preclínica la actividad de ácido valproico, tricostatin A (inhibidor de histonas deacetilasas) y EID1 (inhibidor de EP300), el cual reduce la actividad de PPARg en un modelo de células preadipocíticas 3T3-L1.

Métodos: se realizó transfecciones transitorias con lipofectamina a las células 3T3-L1 y 293. Las células unipotentes 3T3-L1 fueron sometidas a diferenciación con el coctel específico para diferenciación y se les adicionaron los compuestos a concentraciones fisiológicas para las células. Se valoró la expresión de UCP1 mediante Western blot y los experimentos se realizaron por triplicado.

Resultados: se observó que el efecto de tricostatin A fue mayor que el del ácido valproico en actividad lipolítica, no obstante ambos compuestos ejercen una efecto aditivo sobre la actividad de EID1 en la diferenciación de la célula adiposa. EID1 es capaz de estimular la actividad de proteína UCP1, cuya expresión es propia del adipocito marrón.

Conclusiones: EID1 es una proteína que puede ser referente para inducir una célula adiposa calorigénica más activa, reduciendo la acumulación de lípidos en célula grasa. El efecto de ácido valproico y tricostatin A pueden servir de parámetro para la búsqueda de nuevos planes terapéuticos dirigidos a la obesidad. (Acta Med Colomb 2012; 37: 125-130).

Palabras claves: diferenciación adipocito, EID1, tricostatin A, ácido valproico, UCP1.

\begin{abstract}
Objective: obesity has increased worldwide, but there are currently few novel therapeutic options and the tendency to invasive procedures for the therapy of obesity and diabetes mellitus 2 are still an important risk. In order to assess the effect of compounds on the fat cell activity, we studied preclinically the activity of valproic acid, tricostatin A (histone deacetylase inhibitor) and EID1 (EP300 inhibitor) which reduces the activity of PPAR g, in a model of preadipocyte 3T3-L1 cells.

Methods: transient transfections were performed with Lipofectamine in 3T3-L1 and 293 cells. Unipotent 3T3-L1 cells underwent differentiation with the specific cocktail and the compounds were added to cells in physiological concentrations. We assessed the UCP1 expression through western blot, and the experiments were performed in triplicate.

Results: we observed that the effect of tricostatin A was higher than that of the valproic acid in regard to lipolytic activity; however, both compounds exert an additive effect on EID1 activity in adipose cell differentiation. EID1 is able to stimulate the activity of protein UCP1, whose expression is characteristic of brown adipocyte.

Conclusions: EID1 is a reference protein to induce in the adipose cell higher caloric activity, reducing the accumulation of lipids in the adipocyte. The effect of valproic acid and tricostatin A can serve as a parameter for the search of new targeted therapeutic plans for obesity. (Acta Med Colomb 2012; 37: 125-130)
\end{abstract}

Keywords: adipocyte differentiation, EID1, trichostatin A, valproic acid, UCP1.
Dres. Diana Vargas y Luis Celis: Centro de Investigación Biomédica, Universidad de La Sabana. Chía (Cundinamarca); Dr. Fernando Lizcano: PhD, Centro de Investigación Biomédica, Universidad de La Sabana. Fundación Cardio-Infantil IC. Bogotá, D.C. (Colombia).

Correspondencia. Dr. Fernando Lizcano L. Facultad de Medicina. Universidad de La Sabana. Campus Universitario de Puente del Común. Chía. Cundinamarca. Colombia.

E-mail: fernando.lizcano@unisabana.edu.co Recibido: 17/IV/2012 Aceptado: 31/VIII/2012 


\section{Introducción}

La obesidad es un trastorno metabólico y nutricional con serias consecuencias para la salud. Se reconoce el alto riesgo que representa la obesidad para la incidencia de varias enfermedades metabólicas: hipertensión arterial, enfermedad isquémica coronaria, accidentes cerebrovasculares, diabetes mellitus tipo 2 y ciertas formas de cáncer (1). Por tal motivo, el estudio de la función de nuevos reguladores de la diferenciación y proliferación de la célula adiposa es una posible opción terapéutica para la obesidad (2). Dado el escaso número de compuestos que en la actualidad se utilizan para la terapia de la obesidad y que las terapias que modifican los centros hipotalámicos del hambre y saciedad corren un riesgo por los efectos secundarios (3). Una alternativa plausible es dirigir la terapia directamente hacia el adipocito. Algunos compuestos que tiene propiedad de modificar la estructura de la cromatina han demostrado algunos efectos prometedores sobre el metabolismo de la célula grasa (4).

El ácido valproico (VPA) es un fármaco antiepiléptico que impide la aparición de las crisis convulsivas y es un estabilizador del estado de ánimo para el tratamiento del trastorno bipolar y cefaleas crónicas. El VPA presenta efectos secundarios tales como: sedación y cambios en el peso. En estudios in vitro VPA puede reducir el contenido de triglicéridos y modificar la diferenciación del preadipocito (5). Aunque algunos pacientes que toman la medicación pueden tender a incrementar de peso, es posible que los mecanismos de acción de este medicamento tengan un espectro amplio que incluya una acción orexígena en el centro del hambre y saciedad en el hipotálamo (6). El tricostatin A (TSA) es un compuesto orgánico que sirve como antibiótico y antifúngico, que inhibe selectivamente la clase I y II de las histonas de acetilasas (HDAC) (7). El TSA promueve la apoptosis en células cancerosas, retardando la progresión del cáncer y su actividad represora de la transcripción depende del tipo celular $(8,9)$. El papel de la actividad HDAC en la diferenciación del adipocito no está bien definido. Sin embargo, algunos trabajos han estudiado la inhibición en la activación transcripcional de los genes adipogénicos in vitro, incluidas la proteína potenciadora de unión a CCAAT (C/EBP), el receptor de proliferación del peroxisome (PPARg), y la proteína de unión regulada por elementos esteroides SREBP (10).

En los últimos años hemos trabajado con la proteína EID1 (EP300 inhibitor of differentiation 1) descrito inicialmente como inhibidor de diferenciación muscular $(11,12)$. No obstante, nuestras observaciones han evidenciado que puede inhibir la célula preadiposa y reducir la cantidad de lípidos acumulados (13). El mecanismo mediante el que puede ejercer esta función no esta absolutamente determinado pero teóricamente puede inhibir la actividad de PPARg y una posible inhibición adicional de la proteína del retinoblastoma $(\mathrm{pRb})$ que posee un papel importante en las fases iniciales de la diferenciación de la célula adiposa (14).
En el presente trabajo valoramos el papel de VPA, TSA y EID1 sobre células preadiposas. Nuestras observaciones evidencian que la actividad lipolítica de EID1 es incrementada con la adición de estos dos inhibidores de las histonas acetilasas. Es posible que el papel de EID1 sobre la proteína del retinoblastoma quien ejerce su función en relación con la unión con proteínas histonas deacetilasas puede ser el factor clave en la función de EID1. Consideramos que la utilización de compuestos que ejerzan una influencia directa sobre la célula grasa podría ser un determinante importante para terapias contra la obesidad.

\section{Cultivo celular y diferenciación}

\section{Material y métodos}

Se utilizaron las líneas celulares 3T3-L1 y U2OS provenientes de la colección ATCC (American Tissue Culture Collection). Las células fueron mantenidas en medio de Dulbecco's modificado de Eagle's (DMEM) con suero fetal bovino inactivado (FBS) al $10 \%$ y penicilina/estreptomicina al $1 \%$, e incubadas a $37^{\circ} \mathrm{C}$ y $5 \%$ de $\mathrm{CO}_{2}$.

Para la diferenciación de 3T3-L1, dos días después de que las células alcanzaron una confluencia de $100 \%$, se agregó un coctel para inducir la diferenciación que contenía dexametasona $1 \mu \mathrm{M}$, metil isobutil xantina $0.5 \mathrm{mM}$ y rosiglitazona a $1 \mu \mathrm{M}$. Luego de 48 horas se cambió el medio de cultivo y el coctel de diferenciación por solo el medio de cultivo. Posteriormente se cambió el medio DMEM cada dos días. El VPA $1 \mathrm{mM}$, TSA 5nM fueron diluidos en Me2SO $<0.1 \%$ y adicionados hasta el día ocho de la diferenciación. Finalmente se realizaron observaciones a los $0,2,4,6$, días después de la aplicación del coctel de diferenciación. Las células son teñidas con rojo aceite y cuantificadas como lo describen Kasturi y Joshi (15). Se realizó una extracción de lípidos con $5 \mathrm{~mL}$ de isopropanol para luego leer la absorbancia del extracto a $510 \mathrm{~nm}$.

\section{Plásmidos}

Vectores de expresión que contenían PPARg se hallaban pSV. SPORT1 en el sitio SalI. El elemento de respuesta de PPARg se hallaba subclonado en PPRE-LUC) PPRE derivado del gen AP2 y fueron obtenidos del Dr. Bruce Spiegelman (Dana Farber Institute) (16). EID1 y el mutante $\triangle$ EID1 fueron creados por la subclonación en los sitios de restricción BamHI and EcoRI en el plásmido pcDNA3-T7 (17); $\triangle$ EID1 es un mutante que carece de los residuos 53-63 y $92-115$, los que componen el dominio acídico que facilita la interacción con el cofactor p300.

\section{Transfecciones transitorias}

Las células fueron cultivadas en discos de 12 pozos y cuando presentaban una confluencia de $70 \%$ fueron transitoriamente transfretadas mediante Lipofectamine ${ }^{\mathrm{TM}} 2000$ (Invitrogen) con la siguiente concentración de los plásmidos: Plásmido reportero ligado a luciferasa, PPRE-Luc $(0.85$ $\mu \mathrm{g} /$ pozo), vector de expresión con PPARg (0.2 $\mu \mathrm{g} /$ pozo $)$ y 
concentraciones similares para EID1 y $\Delta$ EID1. La cantidad total de $\mathrm{ADN}$ fue estandarizada para todos los experimentos agregando la cantidad correspondiente de pcDNA3.1 en los pozos correspondientes. El control de eficiencia de la transfección fue realizado con el plásmido CMV-bGal a una concentración de $0.5 \mathrm{mg} /$ pozo.

Veinticuatro horas luego de la transfección el medio fue cambiado por DMEM suplementado con $10 \%$ de carbón activado y resinas de intercambio iónico. Se disolvió $1 \mu \mathrm{M}$ de rosiglitazona en dimethyl sulfoxide (DMSO) y adicionado a las células. Una concentración de $0.2 \%$ de DMSO sólo fue utilizado como control. Luego de $24 \mathrm{~h}$, las células fueron obtenidas de los pozos de cultivo mediante el buffer Triton X-100, y se realizó la medición de luciferasa y b-galactosidasa. Para la valoración del efecto de sobre expresión de EID1 en la diferenciación de los adipocitos las células fueron crecidas hasta obtener una confluencia del $90 \%$ y transfectada con $1.6 \mathrm{mg}$ de EID1 in $4 \mathrm{~mL}$ of Lipofectamine ${ }^{\mathrm{TM}} 2000$ (Invitrogen). pcDNA3.1 fue utilizado como control. $48 \mathrm{~h}$ luego de la transfección las células fueron lisadas y se obtuvo las proteínas. Todos los experimentos fueron realizados por triplicados.

\section{Análisis Western Blot}

Los cultivos celulares de 3T3-L1 fueron lisados con 100 $\mathrm{mL} /$ pozo del buffer RIPA (1X PBS, $1 \%$ Nonidet P-40, $0.1 \%$ SDS and $7 \mathrm{mg} / \mathrm{mL}$ de un inhibidor de proteasas, Roche). Luego de centrifugación, $100 \mathrm{mg}$ del total de proteínas fueron mezcladas con un volumen similar del buffer de las muestras y se las proteínas se desnaturalizaron a $95^{\circ} \mathrm{C}$ durante $3 \mathrm{~min}$. Se realizó una electroforesis en gel de SDSpoliacrilamida al $8 \%$ y las muestras fueron trasladas a una membrana de nitrocelulosa. La membrana fue bloqueada con leche al 5\% disuelta a en PBS conteniendo 0.1\% (v/v) Tween 20 (PBS-T) durante $1 \mathrm{~h}$ temperatura ambiente. La membrana fue incubada con el anticuerpo UCP1 (Rabbit anti-mouse) a una dilución durante la noche 1:1000 (Millipore $A B$ 3036). Luego de cuatro lavados con PBS, la membrana fue incubada durante $1 \mathrm{~h}$ con un segundo anticuerpo conjugado con HRP-anti-Rabbit (1:1000). Una solución ECL solución (Amersham) fue aplicada a la membrana y las imágenes de las bandas fueron capturadas mediante autorradiografía de acuerdo a las instrucciones del fabricante.

\section{Análisis estadístico}

Se realizó la prueba de análisis de varianza (Anova) y las diferencias fueron consideradas estadísticamente significativas cuando el valor de la media con error estándar fue de $p<0.05$. Dado que en los estudios de transfección se realizó un cotransfección con $\beta$-galactosidasa, permite normalizar los datos y evita la dispersión de los resultados. $\beta$-galactosidasa cuenta con un promotor diferente al vector con el reportero con lo cual no interfiere con los resultados. Se utilizó el programa StatView (versión original), en las cajas de 12 pozos cada pozo contiene $\pm 5 \times 10^{5}$ células considerando cada célula un sujeto de estudio, se realiza la comparación de tres estudios diferentes realizados por triplicado. Se utilizó la $t$ de Student para comparación de dos tratamientos los cuales fueron realizados por triplicado en tres diferentes experimentos.

\section{Resultados}

Inicialmente realizamos transfecciones transitorias para valorar la actividad de PPARg en estimular la transcripción y el efecto de la proteína EID1 sobre esta actividad. Se observó que PPARg produce un aumento significativo en la transcripción de un gen que presenta elementos de respuestas para PPARg (PPRE) en este caso AP2, el cual a su vez es reducido en forma significativa al adicionar EID1. Cuando la transfección se realiza con el mutante de $\Delta$ EID1 que reduce la unión con el cofactor p300 el efecto de la proteína EID1 se halla ausente (Figura 1). Dado el papel relevante que tiene PPARg en el proceso de diferenciación de la célula adiposa valoramos el efecto de la proteína EID1 sobre la diferenciación de preadipocitos derivados de rata (3T3-L1), observando una reducción significativa en la capacidad de estas células de acumular triglicéridos en la presencia de E1D1 (Figura 2).

Al valorar los hechos previos consideramos evaluar diferentes moléculas que están presente en la célula adiposa y determinar si la adición de EID1 modifica su expresión. Un factor que podría contribuir a especificar esta evaluación, es el hallazgo previo de la unión e inhibición de EID1 sobre la proteína del retinoblastoma (pRb). pRB además de estar involucrada en la patogénesis de varios tipos de cáncer, media en fases iniciales de la diferenciación de células mesenquimales la decisión de las células progenitoras de continuar el linaje blanco o el de célula adiposa marrón. Por tal motivo entre otras proteínas valoramos UCP1 (proteína desacopladora 1), la cual tiene una papel relevante en

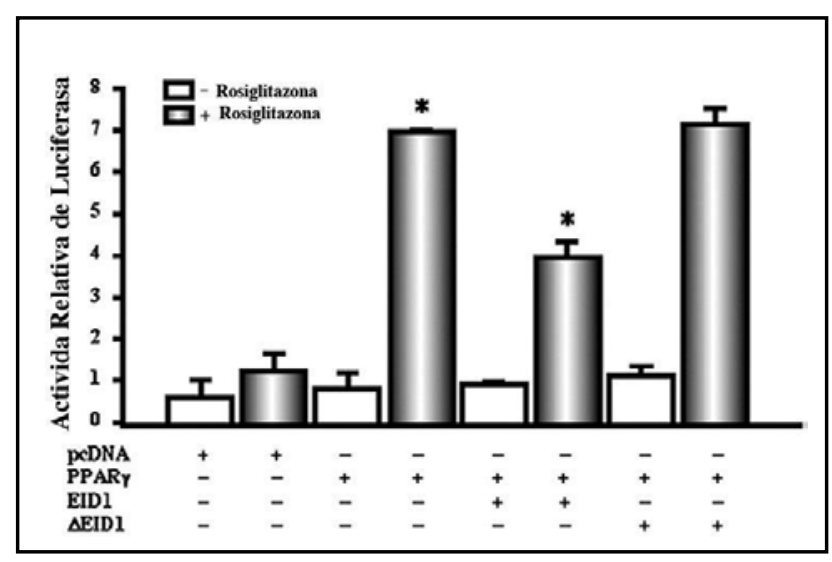

Figura 1. EIDlreduce la función de PPARg y la acumulación de triglicéridos en preadipocitos. Transfecciones transitorias en líneas celulares 293 fueron realizadas con plásmidos reportero PPRE y vectores de expresión en quimera con PPARg, EIDI y el mutante $\triangle E I D I$. Las células fueron lisadas después de 48 h y la determinación de luciferasa y $\beta$-galactosidasa fueron valoradas utilizando un sistema de luminiscencia para luciferasa. Los valores de luciferasa fueron normalizados con los de $\beta$-galactosidasa. Los datos son expresados como la media $\pm S E$ de tres experimentos independientes, fueron analizadas mediante $t$ de Students $y$ un valor de diferencia significativa $(p<0.05)$ es indicado por asterisco. 


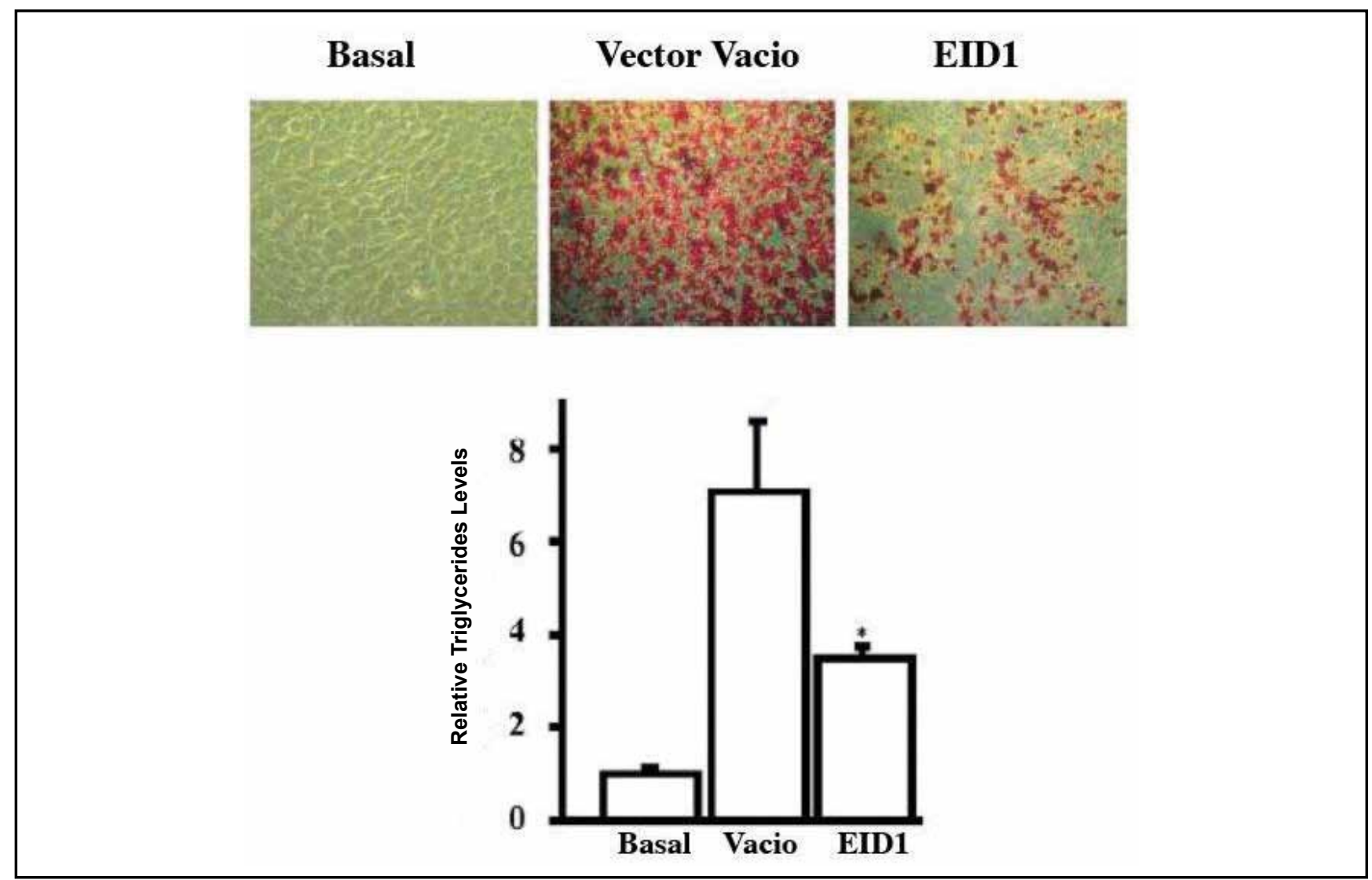

Figura 2. Células preadipocíticas 3T3-LI fueron transfectada con EID1 y se llevó a cabo el proceso de diferenciación, con el coctel de transfección. Luego de 48 horas de transfección, valoró la cantidad de acumulación de triglicéridos de acuerdo con la metodología descrita en el texto. La reducción de la acumulación de triglicéridos al adicionar EID1 se reduce en forma significativa en comparación con un vector que no tiene ningún gen que interfiera con el proceso de diferenciación y está denominado como vector vacío. La figura es representativa de tres experimentos diferentes realizados por triplicado. Los resultados con expresados como la media de \pm S.E. * $p<0,001$.

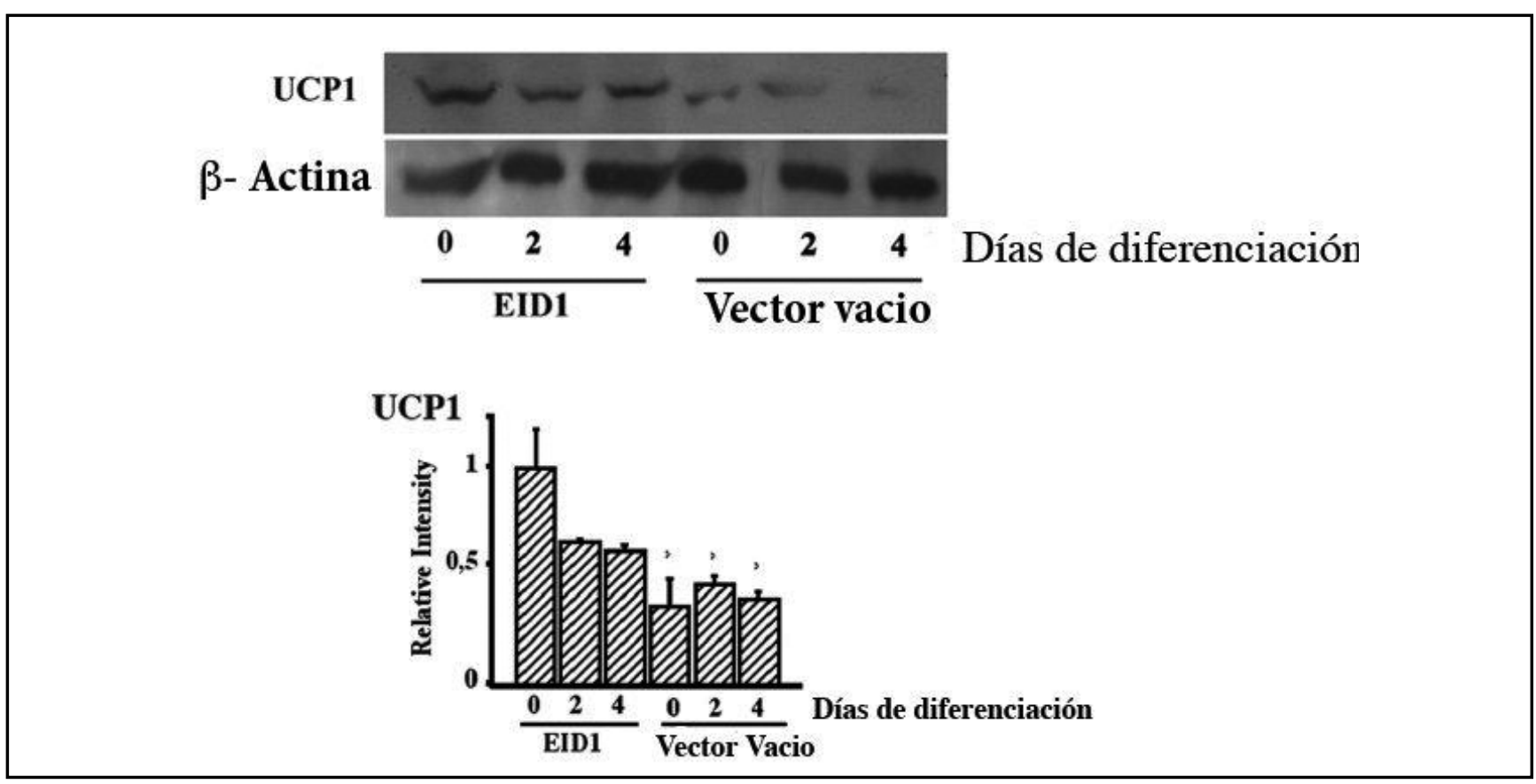

Figura 3. Células de preadipocitos 3T3-L1 fueron transfectadas de acuerdo con los parámetros seguidos en (Fig.1) y la expresión de UCP1 fue analizada por Western Blot en los días indicados, (el día 0 es considerado $48 \mathrm{~h}$ luego de la transfección). El análisis cuantitativo fue realizado mediante un análisis densitométrico de los Western Blot de tres experimentos independientes. El estándar de comparación fue realizado en condición basal el día 0 . Los resultados con expresados como la media de \pm S.E. *p $<0.05$ comparando la presencia o ausencia de EID1. 
el adipocito marrón y no se halla en el adipocito blanco. Al realizar la transfección de los preadipocitos con EID1 observamos un aumento significativo de UCP1 (Figura 3).

La actividad de $\mathrm{pRb}$ en parte cuenta con la ayuda de las histonas acetilasas (HDAC), que reducen la actividad de los genes mediante una modificación de la cromatina. Al utilizar unos compuesto conocidos que tienen efecto inhibidor de HDAC como TSA y VPA observamos una leve reducción en la acumulación de triglicéridos, sin embargo ambos compuestos tiene un efecto aditivo a EID1 sobre la acumulación de triglicéridos en la célula adiposa (Figura 4).

\section{Discusión}

En el presente estudio hemos observado que la reducción de acumulación de lípidos ejercido por la proteína EID1 es aumentado por la actividad de VPA y tricostatin A. La reducción de lípidos ejercida por EID1 es concomitante con un aumento de marcadores de actividad calórico, propios de los adipocitos de origen marrón. La proteína desacopladora UCP1, la cual cumple un papel importante en la producción de calor y es expresada especialmente en el tejido adiposo marrón es aumentado por EID1. Aunque varios estudios han descrito el papel de EID1 en células musculares su papel en la célula grasa son escasos $(11,12)$. En consideración a lo observado en la Figura 1, la reducción de la actividad de PPARg puede ser debida a la inhibición del cofactor p300 y como consecuencia responsable en parte de la reducción de la diferenciación del adipocito observado en la Figura 2. (17). No obstante, la actividad de EID1 podría incluir una modulación de la fisiología del adipocito en fases previas, durante el proceso de diferenciación de adipocito blanco o adipocito marrón (18). Aunque existe evidencia que el adipocito marrón puede tener un origen adicional desde otras líneas progenitoras, la proteína del retinoblastoma tiene una papel fundamental en este proceso (19). Estudios en ratones han demostrado que la deficiencia de $\mathrm{pRb}$ previene a la adiposidad y se acompaña de una alta expresión de UCP1 a niveles similares a los observados en las células adiposas marrones (20). En el presente estudio el aumento de la expresión de UCP1 (Figura 3) nos lleva a teorizar que el efecto de EID1 incluye una reducción de la actividad de pRB. Parte de la actividad de pRb es efectuada a través de la unión con las proteínas deacetilasas de histonas, consideramos que la inhibición efectuada por VPA y TSA sobre estas enzimas puede ser responsable del efecto de reducción de la acumulación de triglicéridos observado en el presente estudio (Figura 4). Hay evidencia clínica de la presencia de adipocito marrón en la persona adulta, incluso las personas que presentan un mayor sobrepeso tienden a presentar menor cantidad de estos adipocitos cuando se realiza el estímulo con frío $(21,22)$.

Hay una gran cantidad de grupos de investigación básicos y clínicos que están procurando la búsqueda de compuestos biológicos o químicos que puedan estimular la presencia de adipocitos marrones en el adulto. Esta podría ser una alternativa terapéutica prometedora para reducir las consecuencias de una pandemia que ha incrementado las enfermedades que inducen riesgo cardiovascular.

\section{Agradecimientos}

Los autores del presente trabajo quieren dar especial agradecimiento a la Dra. Elena Groot por la colaboración incondicional durante la realización del trabajo. También al Dr. Iván Martínez y Carolina Romero por la discusión y el aporte intelectual. Este trabajo ha sido financiado mediante el proyecto 123051929210 de Colciencias.

\section{Declaración de fuentes de financiación y conflictos de interés}

La totalidad del presente trabajo fue financiado mediante concurso interno de la Universidad de La Sabana. La dirección de Investigación de la Universidad de La Sabana que mediante evaluación de la facultad de medicina apoyó financieramente este trabajo con el proyecto MED-134-2010.

No hay declaración de conflictos de interés de ninguno de los autores con las empresas productoras de los compuestos que se evaluaron en el presente trabajo.

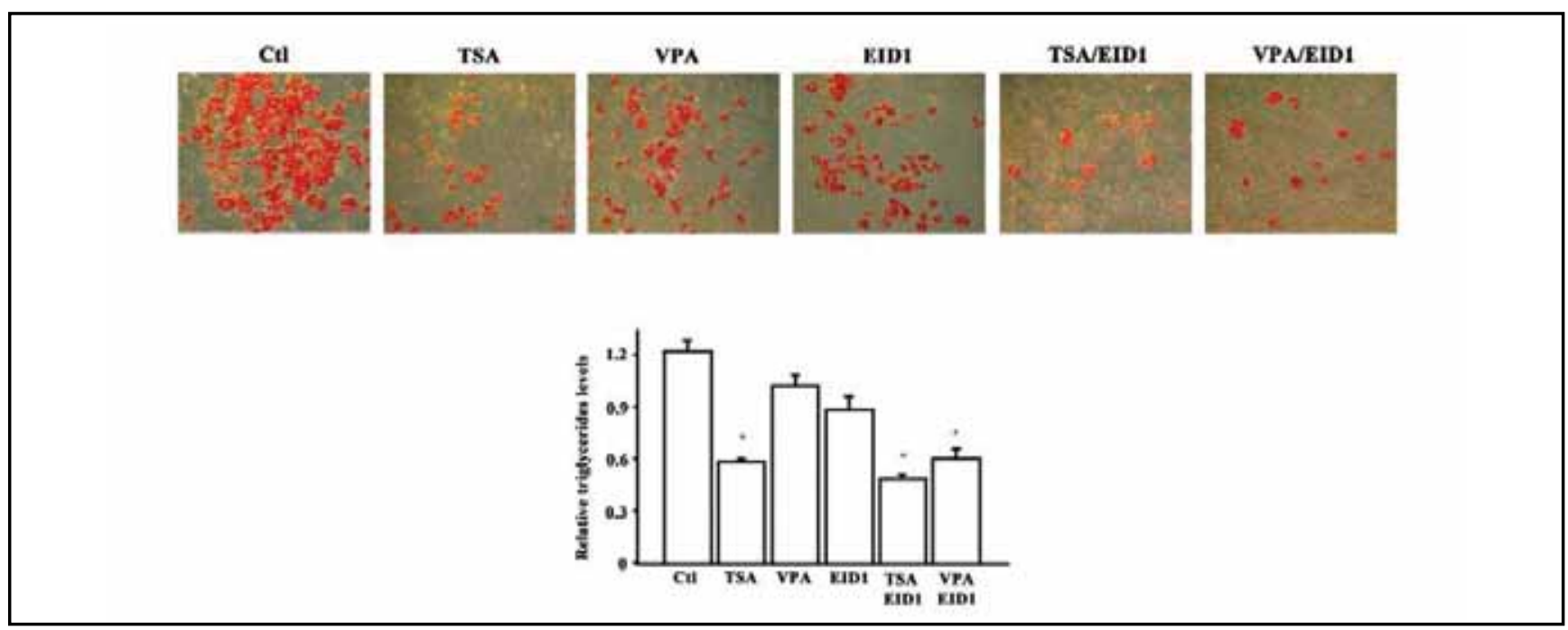

Figura 4. Células preadipocíticas 3T3-L1 fueron sometidas a diferenciación y se les adicionó Tricostatin Aó Ac. Valproico (VPA) en concentraciones descrita en el texto. Cuando fueron transfectadas con EIDI los componentes fueron adicionados luego de 48 h de la transfección. Los resultados con expresados como la media de $\pm S$.E. * $p<0.05$ comparando la presencia o ausencia de EID1. 


\section{Referencias}

1. Popkin BM, Adair LS, Ng SW. Global nutrition transittion and the pandemic of obesity. Nutr Rev 2012; 70: 3-21.

2. Knight JA. Diseases and disorders associated with excess body weight. Ann clin Lab Sci 2011; 41: 107-121.

3. van de Woestijne AP, Monajemi H, Kalkhoven E, Visseren FL. Adipose tissue dysfunction and hypertriglyceridemia: mechanisms and managment. Obes Rev 2011; 12: 829-840.

4. Farmer SR. Transcriptional control of adipocyte formation. Cell Metab 2006; 4: 263-273.

5. Lagace DC, Nachtigal MW. Inhibition of histone deacetylase activity by valproic acid blocks adipogenesis. J Biol Chem 2004; 279: 18851-18860.

6. Jallon P, Picard F. Bodyweight Gain and Anticonvulsants: A Comparative Review. Drug Saf 2001; 24: 969-978.

7. Vanhaecke T, Papeleu P, Elaut G, Rogiers V. Trichostatin A-like hydroxamate histone deacetylase inhibitors as therapeutic agents: toxicological point of view. Curr Med Chem 2004; 11: 1629-1643.

8. Lizcano F, Koibuchi N, Fukuda H, Dangond F, Chin WW. Cell type-specific roles of histone deacetylase in TR ligand-independent transcriptional repression. Mol Cell Endocrinol 2001; 172: 13-20.

9. Drummond DC, Noble CO, Kirpotin DB, Guo Z, Scott GK, Benz CC. Clinical development of histone deacetylase inhibitors as anticancer agents. Annu Rev Pharmacol Toxicol 2005; 45: 495-528.

10. Haberland M, Carrer M, Mokalled MH, Montgomery RL, Olson EN. Redundant control of adipogenesis by histone deacetylases 1 and 2. J Biol Chem 2010; 285(19): 14663-14670.

11. MacLellan WR, Xiao G, Abdellatif M, Schneider MD. A novel Rb- and p300-binding protein inhibits transactivation by MyoD. Mol Cell Biol 2000; 20: 8903-8915.
12. Miyake S, Sellers WR, Safran $M$ et al. Cells degrade a novel inhibitor of differentiation with E1A-like properties upon exiting the cell cycle. Mol Cell Biol 2000; 20: 8889-902.

13. Lizcano F, Vargas D. EID1- induces Brown like adipocyte traits in White 3T3-L1 preadipocyte. Biochem Biophys Res Commun 2010; 398: 160-165.

14. Hallenborg P, Feddersen S, Madsen L, Kristiansen K. The tumor suppressors pRB and p53 as regulators of adipocyte differentiation and function. Expert Opin Therap Targets 2009; 13: 235-246.

15. Kasturi R, Joshi VC. Hormonal Regulation of Stearoyl Coenzyme A Desaturase Activity and Lipogenesis during Adipose Conversion of 3T3-Ll Cells. J Biol Chem 1982; 257: 12224-12230.

16. Puigserver P, Spiegelman BM. Peroxisome Proliferator-Activated ReceptorCoactivator 1 (PGC-1): Transcriptional Coactivator and Metabolic Regulator. Endocr Rev 2003; 24: 78-90.

17. Semple R, Chatterjee V and O'Rahilly S.PPARg and human metabolic disease. J Clin Invest 2006; 116: 581-589.

18. Berger JP. Role of PPARgamma, transcriptional cofactors, and adiponectin in the regulation on nutrient metabolism, adipogenesis and insulin action: View from the chair. Int J Obes 2005; 29: S3-S4.

19. Cinti S. Between Brown and White: novel aspects of adipocyte differentiation. Ann Med 2011; 43: 104-115.

20. Dali-Youcef N, Mataki C, Coste A, Messaddeq N, Giroud S, Blanc S, Koeh C, Champy M-F, Chambon P, Fajas L, Metzger D, Schoonjans K, Auwerz J. Adipose tissue-specific inactivation of the retinoblastoma protein protects against diabesity because of increased energy expenditure. Proc Natl Acad Sci 2007; 104: 10703-10708.

21. Virtanen KA, Lidell ME, Orava $\mathbf{J}$ et al. Functional Brown Adipose Tissue in Healthy Adults. $N$ Engl J Med 2009; 360: 1518-1525. 